\title{
Bochner representable operators on Banach function spaces
}

\author{
Marian Nowak ${ }^{1}$
}

Received: 19 September 2017 / Accepted: 20 March 2018 / Published online: 23 March 2018

(C) The Author(s) 2018

\begin{abstract}
Let $\left(E,\|\cdot\|_{E}\right)$ be a Banach function space, $E^{\prime}$ the Köthe dual of $E$ and $\left(X,\|\cdot\|_{X}\right)$ be a Banach space. It is shown that every Bochner representable operator $T: E \rightarrow X$ maps relatively $\sigma\left(E, E^{\prime}\right)$-compact sets in $E$ onto relatively norm compact sets in $X$. If, in particular, the associated norm $\|\cdot\|_{E^{\prime}}$ on $E^{\prime}$ is order continuous, then every Bochner representable operator $T: E \rightarrow X$ is $\left(\gamma_{E},\|\cdot\|_{X}\right)$-compact, where $\gamma_{E}$ stands for the natural mixed topology on $E$. Applications to Bochner representable operators on Orlicz spaces are given.
\end{abstract}

Keywords Banach function spaces - Orlicz spaces - Mixed topologies · Bochner representable operators · Compact operators

Mathematics Subject Classification 47B38 - 46B40 - 46E30 - 47B05

\section{Introduction and preliminaries}

We assume that $\left(X,\|\cdot\|_{X}\right)$ is a real Banach space with the Banach dual $\left(X^{*},\|\cdot\|_{X^{*}}\right)$. Let $B_{X}$ stand for the closed unit ball in $X$. For terminology concerning Riesz spaces and function spaces, we refer the reader to $[1,2,9,10,20]$.

We assume that $(\Omega, \Sigma, \mu)$ is a complete $\sigma$-finite measure space. By $\Sigma_{f}$ we denote the $\delta$-ring of all sets $A \in \Sigma$ with $\mu(A)<\infty$. Let $L^{0}$ denote the corresponding space of equivalence classes of all $\Sigma$-measurable real functions on $\Omega$. Then $L^{0}$ is a

Marian Nowak

M.Nowak@wmie.uz.zgora.pl

1 Faculty of Mathematics, Computer Science and Econometrics, University of Zielona Góra, ul. Szafrana 4A, 65-516 Zielona Gora, Poland 
super Dedekind complete Riesz space, equipped with the $F$-norm topology $\mathcal{T}_{0}$ of convergence in measure on sets of finite measure.

Let $\left(E,\|\cdot\|_{E}\right)$ be a Banach function space, that is, $E$ is an order ideal of $L^{0}$ with $\operatorname{supp} E=\Omega$ and $\|\cdot\|_{E}$ is a Riesz norm. By $\mathcal{T}_{E}$ we denote the topology of the norm $\|\cdot\|_{E}$. For $r>0$, let $B_{E}(r):=\left\{u \in E:\|u\|_{E} \leq r\right\}$. The Köthe dual $E^{\prime}$ of $E$ is defined by:

$$
E^{\prime}:=\left\{v \in L^{0}: \int_{\Omega}|u(\omega) v(\omega)| d \mu<\infty \text { for all } u \in E\right\}
$$

The associated norm $\|\cdot\|_{E^{\prime}}$ on $E^{\prime}$ is defined for $v \in E^{\prime}$ by

$$
\|v\|_{E^{\prime}}:=\sup \left\{\left|\int_{\Omega} u(\omega) v(\omega) d \mu\right|: u \in E,\|u\|_{E} \leq 1\right\} .
$$

Then supp $E^{\prime}=\Omega$ (see [9, Theorem 6.1.5]). The $\sigma$-order continuous dual $E_{c}^{\sim}$ of $E$ separates the points of $E$ and $E_{c}^{\sim}$ can be identified with $E^{\prime}$ through the Riesz isomorphism $E^{\prime} \ni v \mapsto F_{v} \in E_{c}^{\sim}$, where

$$
F_{v}(u)=\int_{\Omega} u(\omega) v(\omega) d \mu \text { for } u \in E
$$

(see [9, Theorem 6.1.1]). The Mackey topology $\tau\left(E, E^{\prime}\right)$ is a locally convex-solid Hausdorff topology with the $\sigma$-Lebesgue property (see [1, Exercise 18, p. 178]). Note that $\tau\left(E, E^{\prime}\right)=\mathcal{T}_{E}$ if and only if $\|\cdot\|_{E}$ is $\sigma$-order continuous.

In addition to these facts, the following assumptions will be in force without futher mention throughout this paper:

(A) $E \subset L_{l o c}^{1}$, i.e., $A \in \Sigma_{f}$ implies $u \mathbb{1}_{A} \in L^{1}$ for all $u \in E$.

(B) $E$ is perfect, i.e., $E=E^{\prime \prime}$ (equivalently, $\|\cdot\|_{E}$ satisfies both the $\sigma$-Fatou property and the $\sigma$-Levy property (see [1, Definition 3.14], [20, § 110 and Theorem 112.2]).

Note that from $(A)$ it follows that for every $A \in \Sigma_{f}, \mathbb{1}_{A} \in E^{\prime}$. Moreover, from $(B)$ it follows that $E$ is $\sigma\left(E, E^{\prime}\right)$-sequentially complete (see [13, Proposition 1.2]). Note that if a subset $H$ of $E$ is $\sigma\left(E, E^{\prime}\right)$-bounded, then $\sup _{u \in H}\|u\|_{E}<\infty$ (see [10, Lemma 1, p. 20], [9, Theorem 6.1.6]). By $\left(E^{\prime}\right)_{a}$ we denote the ideal in $E^{\prime}$ of all elements of order continuous norms, that is,

$$
\left(E^{\prime}\right)_{a}:=\left\{v \in E^{\prime}:\left\|v_{n}\right\|_{E^{\prime}} \rightarrow 0 \text { if }|v(\omega)| \geq v_{n}(\omega) \downarrow 0 \mu-\text { a.e. }\right\}
$$

Then $\|\cdot\|_{E^{\prime}}$ is order continuous if and only if $\left(E^{\prime}\right)_{a}=E^{\prime}$.

Let $L^{0}(X)$ stand for the linear space of $\mu$-equivalence classes of all strongly $\Sigma$ measurable functions $g: \Omega \rightarrow X$. Let

$$
E^{\prime}(X)=\left\{g \in L^{0}(X):\|g(\cdot)\|_{X} \in E^{\prime}\right\} .
$$


Definition 1.1 A bounded linear operator $T: E \rightarrow X$ is said to be Bochner representable, if there exists $g \in E^{\prime}(X)$ such that

$$
T(u)=\int_{\Omega} u(\omega) g(\omega) d \mu \text { for } u \in E .
$$

\section{Bochner representable operators on Banach spaces}

It is known that every Bochner representable operator $T: L^{1} \rightarrow X$ (where $\mu(\Omega)<$ $\infty)$ maps relatively $\sigma\left(L^{1}, L^{\infty}\right)$-compact sets onto relatively norm compact sets in $X$ (see [5, Lemma 11, pp. 74-75]). Now we extend this result to Bochner representable operators $T: E \rightarrow X$.

Theorem 2.1 Assume that $T: E \rightarrow X$ is a Bochner representable operator. Then the following statements hold:

(i) $T$ is $\left(\tau\left(E, E^{\prime}\right),\|\cdot\|_{X}\right)$-continuous.

(ii) $T$ maps relatively $\sigma\left(E, E^{\prime}\right)$-compact sets in $E$ onto relatively norm compact sets in $X$.

Proof There exists $g \in E^{\prime}(X)$ such that

$$
T(u)=\int_{\Omega} u(\omega) g(\omega) d \mu \text { for } u \in E .
$$

(i) Assume that $x^{*} \in X^{*}$. Then for $u \in E$,

$$
\begin{aligned}
\left|x^{*}(T(u))\right| & =\left|\int_{\Omega} u(\omega) x^{*}(g(\omega)) d \mu\right| \\
& \leq \int_{\Omega}|u(\omega)| \cdot\left\|x^{*}\right\|_{X^{*}}\|g(\omega)\|_{X} d \mu=\left\|x^{*}\right\|_{X^{*}} F_{\|g(\cdot)\|_{X}}(|u|) .
\end{aligned}
$$

It follows that $x^{*} \circ T \in E_{c}^{\sim}$, so $T$ is $\left(\sigma\left(E, E^{\prime}\right), \sigma\left(X, X^{*}\right)\right)$-continuous (see [2, Theorem 9.26]), and hence $T$ is $\left(\tau\left(E, E^{\prime}\right),\|\cdot\|_{X}\right)$-continuous (see [8, Theorem 8.6.1]).

(ii) Assume that $H$ is a relatively $\sigma\left(E, E^{\prime}\right)$-compact subset of $E$. It follows that $H$ is relatively $\sigma\left(E, E^{\prime}\right)$-sequentially compact (see [10, Lemma 11, p. 31]). Since $E$ is $\sigma\left(E, E^{\prime}\right)$-sequentially complete, in view of [13, Proposition 1.1] the set $\left\{u\|g(\cdot)\|_{X}\right.$ : $u \in H\}$ in $L^{1}$ is uniformly integrable. Hence given $\varepsilon>0$ there exist $\Omega_{0} \in \Sigma_{f}$ and $\delta>0$ such that

$$
\begin{aligned}
& \sup _{u \in H} \int_{\Omega \backslash \Omega_{0}}|u(\omega)| \cdot\|g(\omega)\|_{X} d \mu \leq \frac{\varepsilon}{2}, \\
& \sup _{u \in H} \int_{A}|u(\omega)| \cdot\|g(\omega)\|_{X} d \mu \leq \frac{\varepsilon}{2} \text {, whenever } A \in \Sigma \text { and } \mu(A) \leq \delta .
\end{aligned}
$$

Choose a sequence $\left(s_{n}\right)$ of $X$-valued strongly $\mu$-measurable step functions on $\Omega$ such that $s_{n}(\omega) \rightarrow g(\omega) \mu$-a.e. and $\left\|s_{n}(\omega)\right\|_{X} \leq\|g(\omega)\|_{X} \mu$-a.e. for all $n \in \mathbb{N}$ (see 
[7, Theorem 6, p. 4]). Hence $s_{n} \in E^{\prime}(X)$ for $n \in \mathbb{N}$. By the Egorov theorem (see [7, Theorem 42, p. 18]), there exists $A_{0} \in \Sigma$ with $A_{0} \subset \Omega_{0}, \mu\left(\Omega_{0} \backslash A_{0}\right) \leq \delta$ and $\sup _{\omega \in A_{0}}\left\|s_{n}(\omega)-g(\omega)\right\|_{X} \rightarrow 0$. For every $n \in \mathbb{N}$ define an operator $T_{n}: E \rightarrow X$ by

$$
T_{n}(u):=\int_{A_{0}} u(\omega) s_{n}(\omega) d \mu \text { for } u \in E .
$$

Note that for every $n \in \mathbb{N}, T_{n}$ is a bounded operator of finite dimensional range, so that $T_{n}$ is a compact operator. Define an operator $T_{A_{0}}: E \rightarrow X$ by

$$
T_{A_{0}}(u):=\int_{A_{0}} u(\omega) g(\omega) d \mu \text { for } u \in E
$$

Then for $u \in B_{E}(1)$, we have

$$
\begin{aligned}
\left\|\int_{A_{0}} u(\omega)\left(g(\omega)-s_{n}(\omega)\right) d \mu\right\|_{X} & \leq \int_{A_{0}}|u(\omega)| \cdot\left\|g(\omega)-s_{n}(\omega)\right\|_{X} d \mu \\
& \leq\left(\int_{\Omega}|u(\omega)| \mathbb{1}_{A_{0}}(\omega) d \mu\right) \sup _{\omega \in A_{0}}\left\|g(\omega)-s_{n}(\omega)\right\|_{X} \\
& \leq\left\|\mathbb{1}_{A_{0}}\right\|_{E^{\prime}} \sup _{\omega \in A_{0}}\left\|g(\omega)-s_{n}(\omega)\right\|_{X} .
\end{aligned}
$$

Hence $\left\|T_{A_{0}}-T_{n}\right\| \leq\left\|\mathbb{1}_{A_{0}}\right\|_{E^{\prime}} \sup _{\omega \in A_{0}}\left\|g(\omega)-s_{n}(\omega)\right\|_{X}$, and hence $\left\|T_{A_{0}}-T_{n}\right\| \rightarrow$ 0 . It follows that $T_{A_{0}}$ is a compact operator. Since $\sup _{u \in H}\|u\|_{E}<\infty$, we have that $K_{\varepsilon}:=\left\{T\left(\mathbb{1}_{A_{0}} u\right): u \in H\right\}$ is a relatively norm compact set in $X$.

Moreover, since $\mu\left(\Omega_{0} \backslash A_{0}\right) \leq \delta$, for every $u \in H$, by (2.2) we get

$$
\left\|T\left(\mathbb{1}_{\Omega_{0} \backslash A_{0}} u\right)\right\|_{X} \leq \int_{\Omega \backslash A_{0}}|u(\omega)| \cdot\|g(\omega)\|_{X} d \mu \leq \frac{\varepsilon}{2}
$$

i.e., $\left\{T\left(\mathbb{1}_{\Omega_{0} \backslash A_{0}} u\right): u \in H\right\} \subset \frac{\varepsilon}{2} B_{X}$. For every $u \in H$, by (2.1) we have

$$
\left\|T\left(\mathbb{1}_{\Omega \backslash \Omega_{0}} u\right)\right\|_{X} \leq \int_{\Omega \backslash \Omega_{0}}|u(\omega)| \cdot\|g(\omega)\|_{X} d \mu \leq \frac{\varepsilon}{2},
$$

i.e., $\left\{T\left(\mathbb{1}_{\Omega \backslash \Omega_{0}} u\right): u \in H\right\} \subset \frac{\varepsilon}{2} B_{X}$. Hence for every $u \in H$, we have

$$
T(u)=T\left(\mathbb{1}_{\Omega \backslash \Omega_{0}} u\right)+T\left(\mathbb{1}_{\Omega_{0} \backslash A_{0}} u\right)+T\left(\mathbb{1}_{A_{0}} u\right) \in \varepsilon B_{X}+K_{\varepsilon} .
$$

In view of the Grothendieck's compactness criterion (see [4, Exercise 4(iii)]) $T(H)$ is a relatively norm compact set in $X$, as desired.

By $\gamma\left[\mathcal{T}_{E}, \mathcal{T}_{0}\right]$ (in brief, $\gamma_{E}$ ) we denote the natural mixed topology on $E$ in the sense of Wiweger (see [3,11,12,19], for more details). Then $\left.\mathcal{T}_{0}\right|_{E} \subset \gamma_{E} \subset \mathcal{T}_{E}$ and $\gamma_{E}$ is the finest linear topology on $E$ that agrees with $\mathcal{T}_{0}$ on every ball $B_{E}(r), r>0$ (see $[19,2.2 .2]) . \mathcal{T}_{E}$ and $\gamma_{E}$ have the same bounded sets in $E$, and for a sequence $\left(u_{n}\right)$ 
in $E, u_{n} \rightarrow 0$ in $\gamma_{E}$ if and only if $u_{n} \rightarrow 0$ in $\mathcal{T}_{0}$ and $\sup _{n}\left\|u_{n}\right\|_{E}<\infty$ (see [19, Corollary, p. 56 and Theorem 2.6.1]). If, in particular, supp $\left(E^{\prime}\right)_{a}=\Omega$, then $\gamma_{E}$ is a locally convex-solid Hausdorff topology and $\gamma_{E}$ coincides with the mixed topology $\gamma\left[\mathcal{T}_{E},|\sigma|\left(E,\left(E^{\prime}\right)_{a}\right)\right]$ (see [11, Theorem 3.3]). Note that, then $\left(E, \gamma_{E}\right)$ is a generalized DF-space (see [15] for more details). In view of [11, Theorem 3.1], we have:

$$
\left(E, \gamma_{E}\right)^{*}=\left\{F_{v}: v \in\left(E^{\prime}\right)_{a}\right\}
$$

Theorem 2.2 Assume that $\left(E,\|\cdot\|_{E}\right)$ is a Banach function space with the order continuous accociated norm $\|\cdot\|_{E^{\prime}}$ on $E^{\prime}$. Let $T: E \rightarrow X$ be a Bochner representable operator. Then the following statements hold:

(i) $T$ is $\left(\gamma_{E},\|\cdot\|_{X}\right)$-continuous and norm-compact operator.

(ii) $T$ is $\left(\gamma_{E},\|\cdot\|_{X}\right)$-compact, that is, there exists a $\gamma_{E}$-neighborhood $V$ of 0 in $E$ such that $T(V)$ is a relatively norm compact set in $X$.

Proof (i) There exists $g \in\left(E^{\prime}\right)_{a}(X)$ such that

$$
T(u)=\int_{\Omega} u(\omega) g(\omega) d \mu \text { for } u \in E .
$$

For $u \in E$, we have

$$
\|T(u)\|_{X} \leq \int_{\Omega}|u(\omega)|\|g(\omega)\|_{X} d \mu=F_{\|g(\cdot)\|_{X}}(|u|)
$$

where $\|g(\cdot)\|_{X} \in\left(E^{\prime}\right)_{a}$. Using (2.3) we obtain that $T$ is $\left(\gamma_{E},\|\cdot\|_{X}\right)$-continuous. Choose a sequence $\left(s_{n}\right)$ of $X$-valued strongly $\mu$-measurable step-functions on $\Omega$ such that $\left\|s_{n}(\omega)-g(\omega)\right\|_{X} \rightarrow 0 \mu$-a.e. and $\left\|s_{n}(\omega)\right\|_{X} \leq\|g(\omega)\|_{X} \mu$-a.e. and all $n \in \mathbb{N}$ (see [7, Theorem 6, p. 4]). Hence $s_{n} \in\left(E^{\prime}\right)_{a}(X)$ and $\left\|s_{n}(\omega)-g(\omega)\right\|_{X} \leq 2\|g(\omega)\|_{X} \mu$-a.e. for all $n \in \mathbb{N}$. Let $v_{n}(\omega)=\sup _{k \geq n}\left\|s_{n}(\omega)-g(\omega)\right\|_{X}$ for $\omega \in \Omega$. Then $2\|g(\omega)\|_{X} \geq$ $v_{n}(\omega) \downarrow 0 \mu$-a.e., so \|\|$s_{n}(\cdot)-g(\cdot)\left\|_{X}\right\|_{E^{\prime}} \leq\left\|v_{n}\right\|_{E^{\prime}} \rightarrow 0$.

For each $n \in \mathbb{N}$, let $T_{n}: E \rightarrow X$ be a linear operator defined by

$$
T_{n}(u):=\int_{\Omega} u(\omega) s_{n}(\omega) d \mu \text { for } u \in E .
$$

Note that the range of each $T_{n}$ is contained in the span of the finite set of values of $s_{n}$. Therefore $T_{n}$ is compact for each $n \in \mathbb{N}$, and for each $u \in E$,

$$
\begin{aligned}
\left\|\left(T_{n}-T\right)(u)\right\|_{X} & =\left\|\int_{\Omega} u(\omega)\left(s_{n}(\omega)-g(\omega)\right) d \mu\right\|_{X} \\
& \leq \int_{\Omega}|u(\omega)| \cdot\left\|s_{n}(\omega)-g(\omega)\right\|_{X} d \mu \\
& \leq\|u\|_{E} \cdot\|\| s_{n}(\cdot)-g(\cdot)\left\|_{X}\right\|_{E^{\prime}}
\end{aligned}
$$

It follows that $\left\|T-T_{n}\right\| \rightarrow 0$, so $T$ is a compact operator. 
(ii) Since $\left(E, \gamma_{E}\right)$ is a generalized DF-space, it is quasinormable (see [15, p. 422]). Hence in view of (i) by the Grothendieck's classical results (see [15, p. 429]), we obtain that $T$ is $\left(\gamma_{E},\|\cdot\|\right)$-compact.

Remark 2.1 A related result to Theorem 2.2 can be found in [17, Corollary 4.8].

An important class of Banach function spaces are Orlicz spaces (see $[10,16]$ for more details). By a Young function we mean here s convex continuous mapping $\Phi:[0, \infty) \rightarrow[0, \infty)$ that vanishes only at 0 and $\Phi(t) / t \rightarrow 0$ as $t \rightarrow 0$ and $\Phi(t) / t \rightarrow \infty$ as $t \rightarrow \infty$. By $\Phi^{*}$ we denote the Young function complementary to $\Phi$ in the sense of Young.

The Orlicz space

$$
L^{\Phi}:=\left\{u \in L^{0}: \int_{\Omega} \Phi(\lambda|u(\omega)|) d \mu<\infty \text { for some } \lambda>0\right\}
$$

equipped with the topology $\mathcal{T}_{\Phi}$ of the norm:

$$
\|u\|_{\Phi}:=\inf \left\{\lambda>0: \int_{\Omega} \Phi(|u(\omega)| / \lambda) d \mu \leq 1\right\}
$$

is a perfect Banach function space (see $[10,16])$. Then $\left(L^{\Phi}\right)^{\prime}=L^{\Phi^{*}}$ and $\left(L^{\Phi^{*}}\right)_{a}=$ $E^{\Phi^{*}}=\left\{v \in L^{\Phi^{*}}: \int_{\Omega} \Phi(\lambda|v(\omega)|) d \mu<\infty\right.$ for all $\left.\lambda>0\right\}$. In particular, $E^{\Phi^{*}}=L^{\Phi^{*}}$ if $\Phi^{*}$ satisfies the $\Delta_{2}$-condition, i.e., $\Phi^{*}(2 t) \leq d \Phi^{*}(t)$ for some $d>1$ and all $t \geq 0$.

We say that a Young function $\Psi$ increases more rapidly than $\Phi$ (in symbols, $\Phi \prec \Psi$ ) if for an arbitrary $c>0$ there exists $d>0$ such that $c \Phi(t) \leq \frac{1}{d} \Psi(d t)$ for all $t \geq 0$.

If $\Phi \prec \Psi$, then $L^{\Psi} \subset L^{\Phi}$ and by $i_{\Psi}: L^{\Psi} \rightarrow L^{\Phi}$ we denote the inclusion map.

Proposition 2.3 Let $T: L^{\Phi} \rightarrow X$ be a Bochner representable operator. Then for every Young function $\Psi$ with $\Phi \prec \Psi$ the operator $T \circ i_{\Psi}: L^{\Psi} \rightarrow X$ is compact.

Proof Assume that $\Psi$ is a Young function with $\Phi \prec \Psi$. Then by [16, Theorem 5.3.3, p. 171] the closed unit ball $B_{\Psi}(1)$ in $L^{\Psi}$ is relatively $\sigma\left(L^{\Phi}, L^{\Phi^{*}}\right)$-compact in $L^{\Phi}$. Hence according to Theorem $2.1 T\left(B_{\Psi}(1)\right)$ is relatively norm compact, and this means that $T \circ i_{\Psi}: L^{\Psi} \rightarrow X$ is compact.

We say that a Young function $\Phi$ increases essentially more rapidly than another $\Psi$ (in symbols, $\Psi \ll \Phi$ ) if for an arbitrary $c>0, \Psi(c t) / \Phi(t) \rightarrow 0$ as $t \rightarrow 0$ and $t \rightarrow \infty$.

The following characterization of the mixed topology $\gamma_{\Phi}\left(=\gamma\left[\mathcal{T}_{\Phi}, \mathcal{T}_{0}\right]\right)$ on $L^{\Phi}$ will be useful (see [14, Theorem 2.1]).

Theorem 2.4 Let $\Phi$ be a Young function. Then the mixed topology $\gamma_{\Phi}$ on $L^{\Phi}$ is generated by the family of norms $\left\{\left.\|\cdot\|_{\Psi}\right|_{L^{\Phi}}: \Psi \ll \Phi\right\}$.

As a consequence of Theorems 2.2 and 2.4 we have:

Corollary 2.5 Assume that a Young function $\Phi^{*}$ satisfies the $\Delta_{2}$-condition. Let $T$ : $L^{\Phi} \rightarrow X$ be a Bochner representable operator. Then there exists a Young function $\Psi$ with $\Psi \ll \Phi$ such that $T\left(B_{\Psi}(1) \cap L^{\Phi}\right)$ is a relatively norm compact set in $X$. 
Remark (i) The result of Corollary 2.5 was established in a different way in [14, Theorem 2.3].

(ii) For a bounded linear operator $T: L^{\Phi} \rightarrow X$, following [6] one can define its functional norm $\||T|\|$ by $\left\|\left|T\|\mid=\sup \Sigma\| \alpha_{i} T\left(\mathbb{1}_{A_{i}}\right) \|_{X}\right.\right.$, where the supremum is taken over all finite $\Sigma$-partition $\left(A_{i}\right)$ of $\Omega$ and all $\alpha_{i} \in \mathbb{R}$ such that $\left\|\Sigma \alpha_{i} \mathbb{1}_{A_{i}}\right\|_{\Phi} \leq 1$.

Uhl [18, Theorem 1] showed that if $X$ either is reflexive or is a separable dual Banach space and $\Phi$ obeys the $\Delta_{2}$-condition, then every bounded linear operator $T: L^{\Phi} \rightarrow X$ with $\left\||T \||<\infty\right.$ is Bochner representable. If, in addition, $\Phi^{*}$ also obeys the $\Delta_{2}$-condition, then every bounded operator $T: L^{\Phi} \rightarrow X$ with $\|\mid T\| \|<\infty$ is compact (see [18, Corollary 2]).

Open Access This article is distributed under the terms of the Creative Commons Attribution 4.0 International License (http://creativecommons.org/licenses/by/4.0/), which permits unrestricted use, distribution, and reproduction in any medium, provided you give appropriate credit to the original author(s) and the source, provide a link to the Creative Commons license, and indicate if changes were made.

\section{References}

1. Aliprantis, C.D., Burkinshaw, O.: Locally Solid Riesz Spaces with Applications to Economics, 2nd edn. American Mathematical Society, Providence (2003)

2. Aliprantis, C.D., Burkinshaw, O.: Positive Operators. Academic Press, New York (1985)

3. Cooper, J.B.: The strict topology and spaces with mixed topologies. Proc. Am. Math. Soc. 30(3), 583-592 (1971)

4. Diestel, J.: Sequences and Series in Banach Spaces, Graduate Texts in Mathematics. Springer, Berlin (1984)

5. Diestel, J., Uhl, J.J.: Vector Measures (Mathematical Surveys, 15). American Mathematical Society, Providence (1977)

6. Dinculeanu, N.: Vector Measures. Pergamon Press, Oxford (1967)

7. Dinculeanu, N.: Vector Integration and Stochastic Integration in Banach Spaces. A Wiley-Interscience Publication, New York (2000)

8. Edwards, R.E.: Functional Analysis, Theory and Applications. Holt, Rinehart and Winston, New York (1965)

9. Kantorovitch, L.V., Akilov, G.P.: Functional Analysis. Pergamon Press, Oxford (1982)

10. Luxemburg, W.: Banach Functions Spaces. Thesis, Delft (1955)

11. Nowak, M.: Mixed topology on normed function spaces, I. Bull. Pol. Acad. Sci. Math. 36(5-6), 251262 (1988)

12. Nowak, M.: Mixed topology on normed function spaces, II. Bull. Pol. Acad. Sci. Math. 36(5-6), 263-267 (1988)

13. Nowak, M.: Conditional and relative weak compactness in vector-valued function spaces. J. Convex Anal. 12(2), 447-463 (2005)

14. Nowak, M.: Compactness of Bochner representable operators. Positivity 13, 193-199 (2009)

15. Ruess, W.: [Weakly] compact operators and DF-spaces. Pacific J. Math. 98, 419-441 (1982)

16. Rao, M.M., Ren, Z.D.: Theory of Orlicz Spaces. Marcel Dekker, New York (1991)

17. Uhl, J.J.: Vector integral operators. Indag. Math. 32, 463-478 (1970)

18. Uhl, J.J.: On a class of operators on Orlicz spaces. Studia Math. 40, 17-22 (1971)

19. Wiweger, A.: Linear spaces with mixed topology. Studia Math. 20, 47-68 (1961)

20. Zaanen, A.C.: Riesz Spaces II. North-Holland Publishing Company, Amsterdam (1983) 